\title{
Supplemental Tables
}

\begin{tabular}{|c|c|c|c|c|}
\hline \multicolumn{5}{|c|}{ Supplemental Table 1: Primers Used in Cloning of the FGAR-AT } \\
\hline ID & Name & Sequence & $\begin{array}{l}\text { Restriction } \\
\text { Enzyme }\end{array}$ & Plasmid \\
\hline 1 & PurS-F & $\begin{array}{l}\text { GCCATATGTATAAAGTAAAAG } \\
\text { TTTATGTC }\end{array}$ & NdeI & pET-PurS \\
\hline 2 & PurS-R & $\begin{array}{l}\text { CGGGATCCTCACTGTGCGACTA } \\
\text { CCTCCTCAAC }\end{array}$ & BamHI & pET-PurS \\
\hline 3 & QDN & $\begin{array}{l}\text { TCTTTACTCGAGTCAAGCAGTA } \\
\text { GTGACATGAG }\end{array}$ & XhoI & $\begin{array}{l}\text { pET-PurQ-WT } \\
\text { and A128T }\end{array}$ \\
\hline 4 & QUP & $\begin{array}{l}\text { AGTCGCCATATGAAATTTGCGG } \\
\text { TGATTGT }\end{array}$ & NdeI & $\begin{array}{l}\text { pET-PurQ-WT } \\
\text { and A128T }\end{array}$ \\
\hline 5 & Bs-L-F & $\begin{array}{l}\text { GCCATATGTCACTACTGCTTGA } \\
\text { ACCAAGTAAAGAAC }\end{array}$ & NdeI & pET-smPurL \\
\hline 6 & Bs-L-R & $\begin{array}{l}\text { CGCATTGGATCCTTAAGCCTTTG } \\
\text { ATTTCAGCAAGCATGG }\end{array}$ & BamHI & $\begin{array}{l}\text { pET-smPurL } \\
\text { and pDUET-L }\end{array}$ \\
\hline 7 & SL-NcoI & $\begin{array}{l}\text { GCCCATGGCACTACTGCTTGAAC } \\
\text { CAAGTAAAGAAC }\end{array}$ & NcoI & pDUET-L \\
\hline 8 & Q-NdeI & $\begin{array}{l}\text { GCCATATGAAATTTGCGGTGATT } \\
\text { GTGTTACCC }\end{array}$ & NdeI & pDUET-LQ \\
\hline 9 & Q-KpnI & $\begin{array}{l}\text { GGGGTACCTCAAGCAGTAGTGA } \\
\text { CATG }\end{array}$ & KpnI & pDUET-LQ \\
\hline
\end{tabular}




\section{Supplemental Figures}

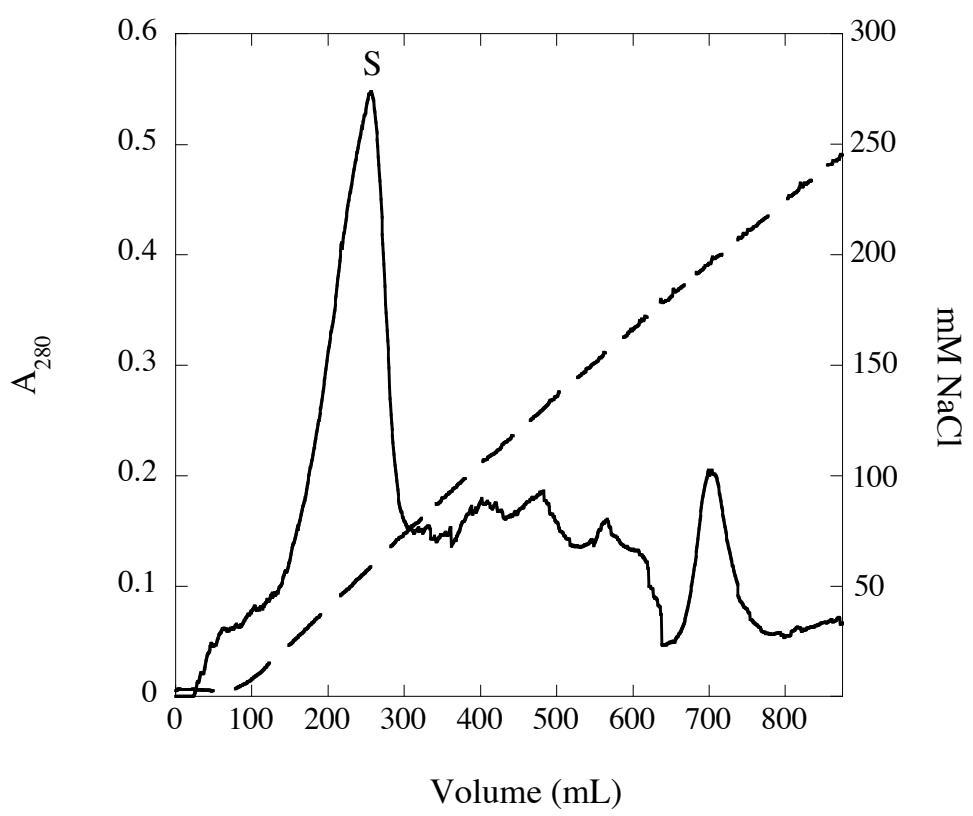

Supplemental Figure 1: DEAE Elution profile of PurS. Elution of PurS (S) is noted. 


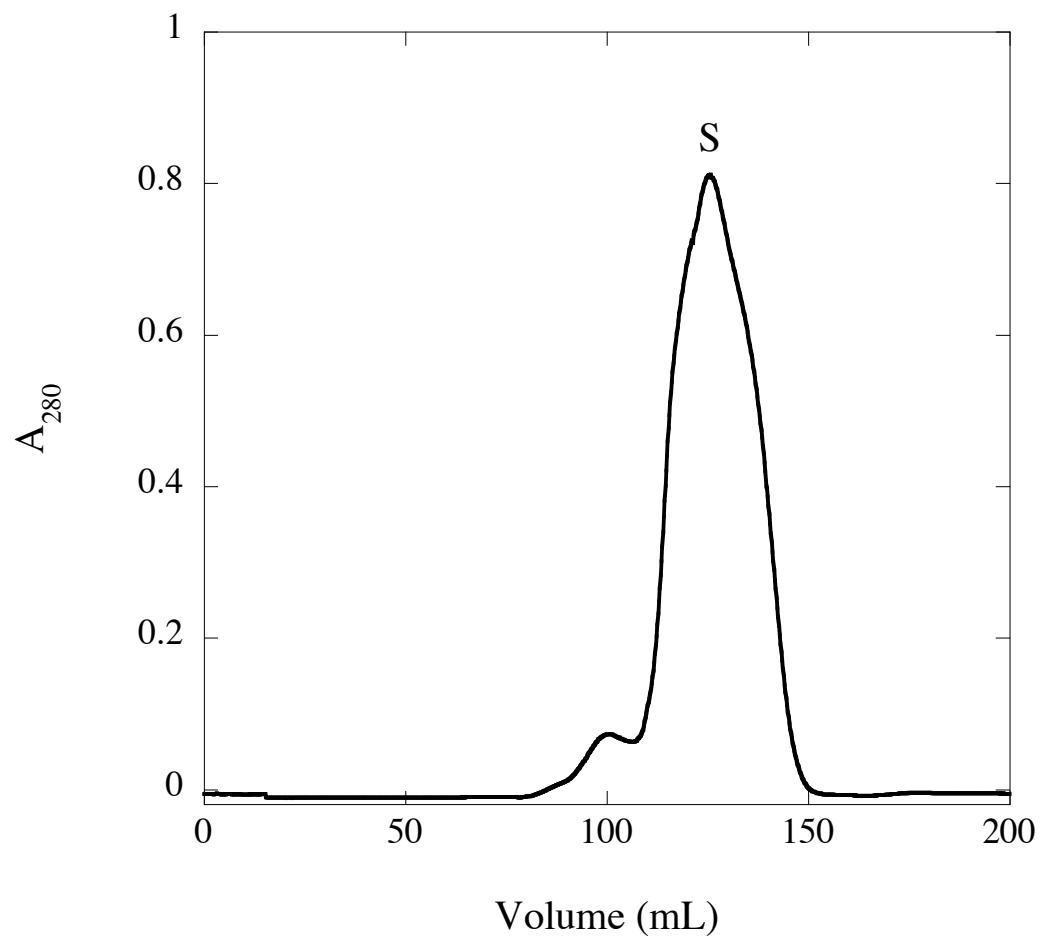

Supplemental Figure 2: Sephacryl S-300 elution profile of PurS. The elution of PurS (S) is noted. 




Supplemental Figure 3: DEAE elution profile of A128T PurQ. Activity ( ) was measured using the coupled GDH assay and is expressed in terms of the change in $\mathrm{A}_{363}$ per min $\times 10$ in order to plot the $\mathrm{A}_{280}$ and relative activity on the same axis. 


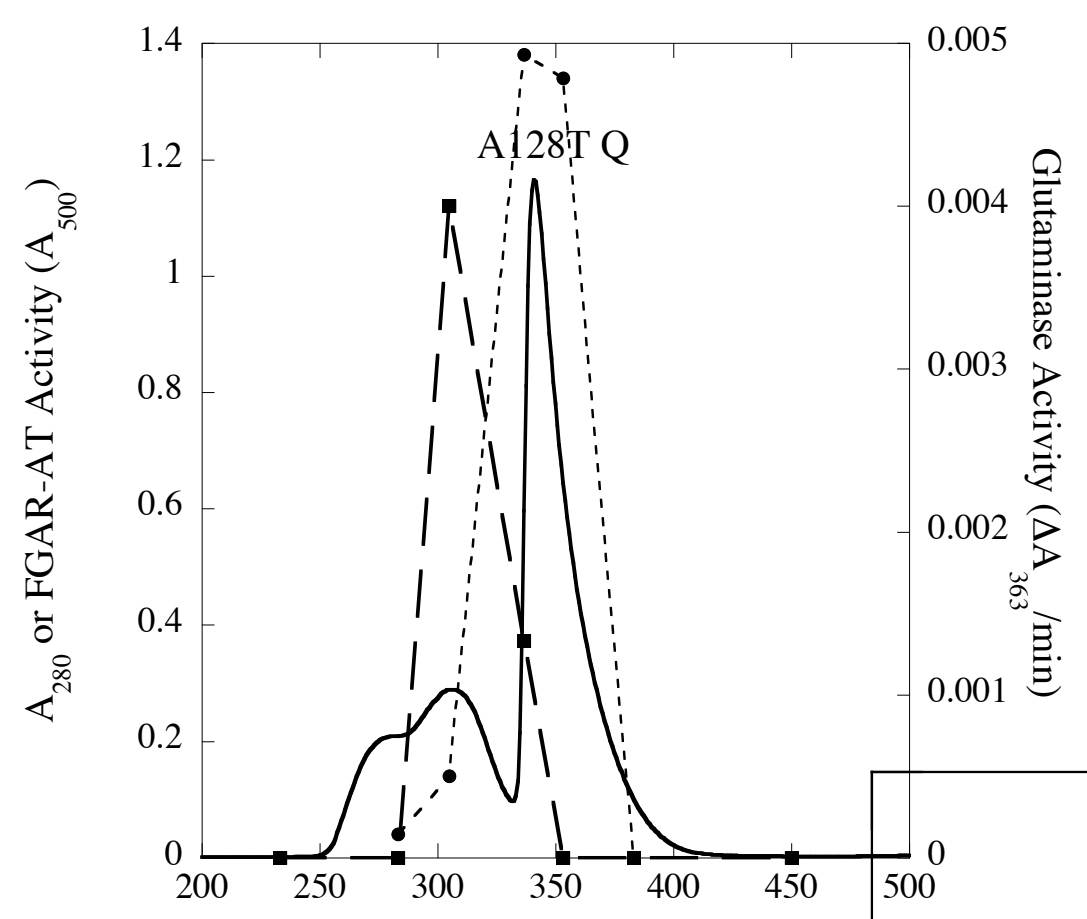

Time (min)

Supplemental Figure 4: Sephacryl S-200 SEC elution profile of A128T PurQ. FGARAT activity ( , dotted line) is represented as $\mathrm{A}_{500}$ from the gln-dependent BrattonMarshall assay. Glutaminase activity (', dashed line) is represented as the change in $\mathrm{A}_{363}$ per min from the GDH-coupled assay. Notice that an endogenous E. coli glutaminase copurified with PurQ. The glutaminase was removed during SEC and eluted at $300 \mathrm{~min}$. PurQ eluted at 350 min. 


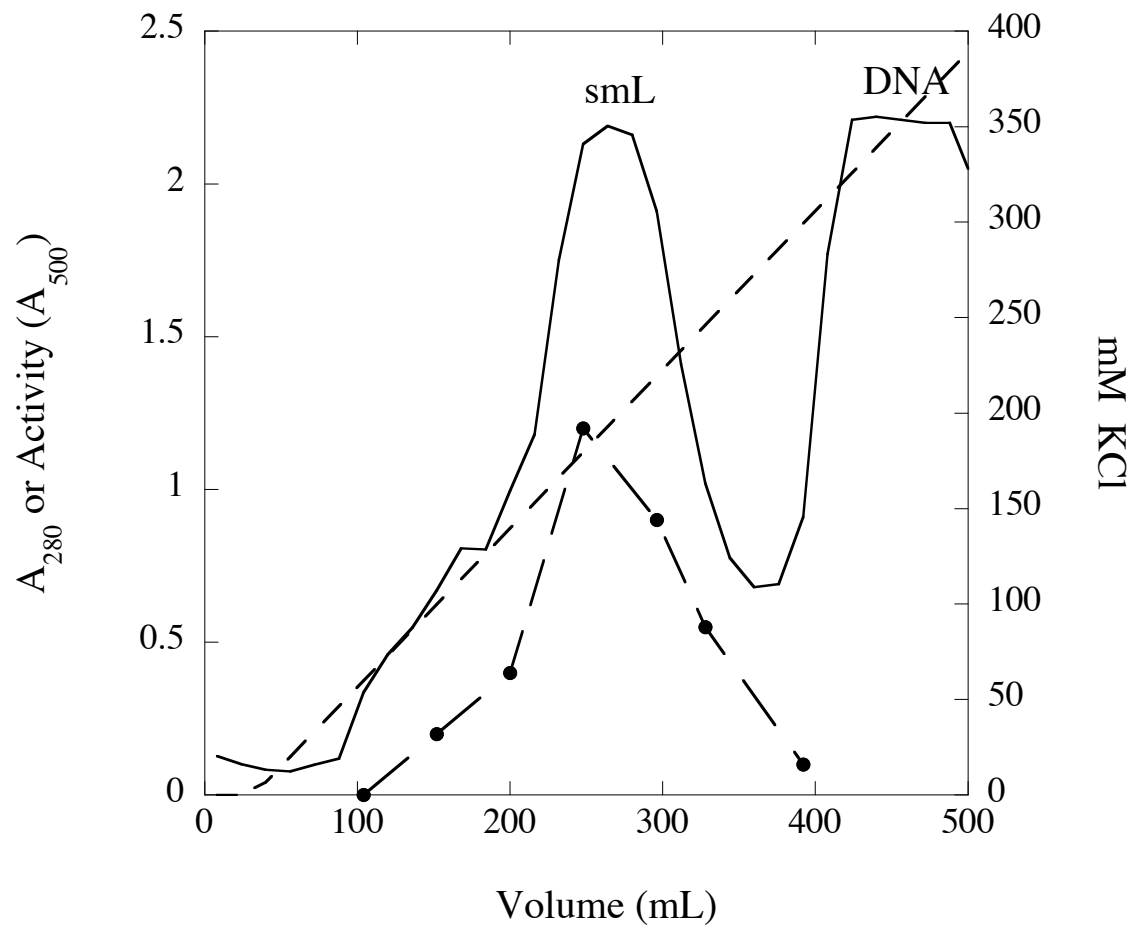

Supplemental Figure 5: DEAE elution profile of smPurL. The activity ( , dashed line) is expressed as $\mathrm{A}_{500}$ from the $\mathrm{NH}_{3}$-dependent Bratton-Marshall assay. Elution of smPurL and cellular DNA is noted. 


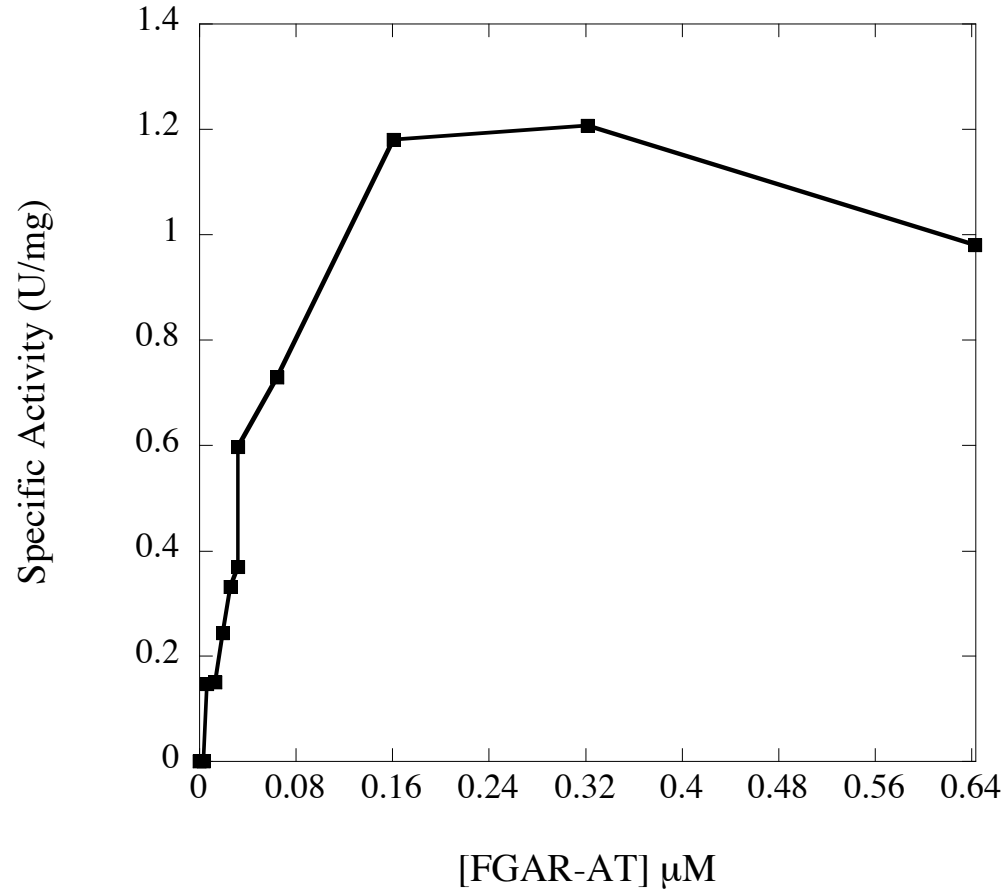

Supplemental Figure 6: Concentration dependence of the specific activity of the FGARAT complex. The FGAR-AT complex was reconstituted as described and diluted into assay buffer at varying concentrations. 


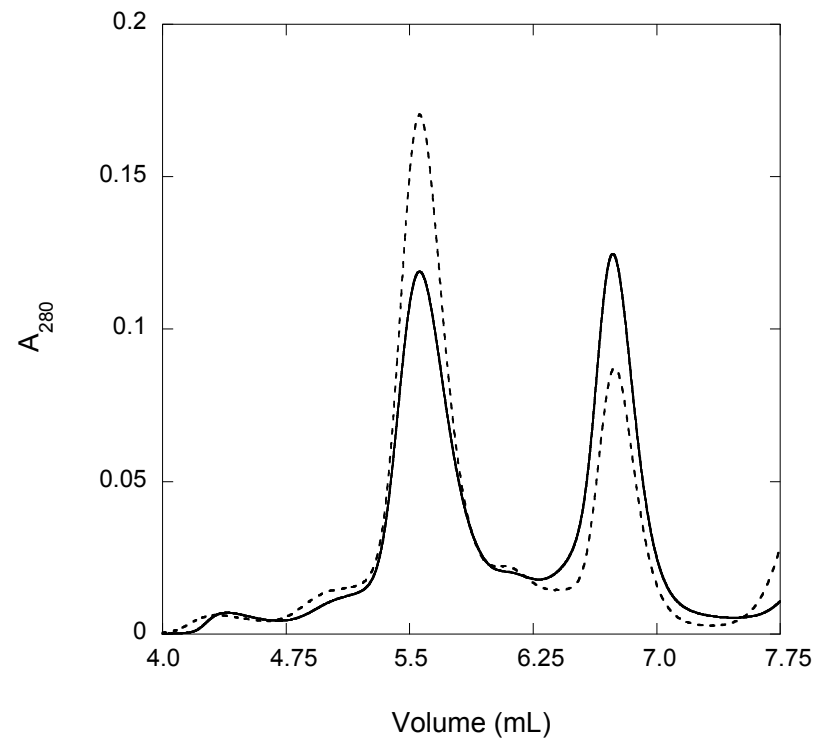

Supplemental Figure 7: Partial complex formation observed with pre-incubation of 2 PurS: 1 smPurL: 1 PurQ with MgADP and glutamine and elution with only glutamine included in the SEC buffer. The solid line shows the FGAR-AT proteins eluted in only SEC buffer. The dotted line shows formation of the FGAR-AT complex initiated by including glutamine in the SEC buffer. 


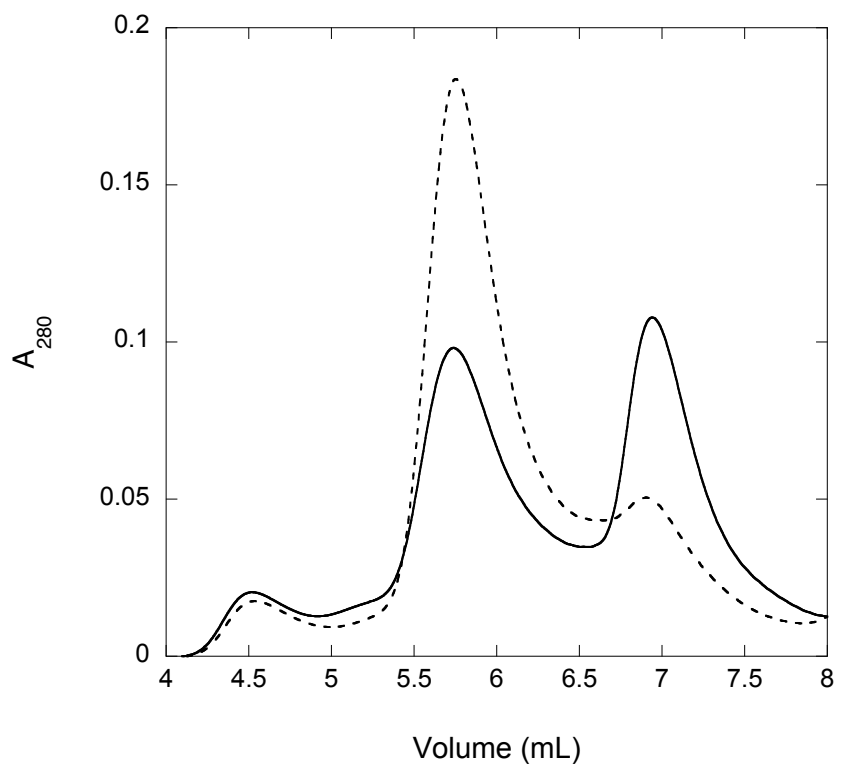

Supplemental Figure 8: Partial 2 PurS: 1 smPurL: 1 PurQ complex formation observed with DON-inactivated PurQ. The solid line shows the 2 PurS; 1 smPurL: 1 PurQ proteins eluted in SEC buffer. The dotted line shows the 2 PurS; 1 smPurL: 1 PurQ proteins eluted in SEC buffer after DON-inactivation of PurQ. 


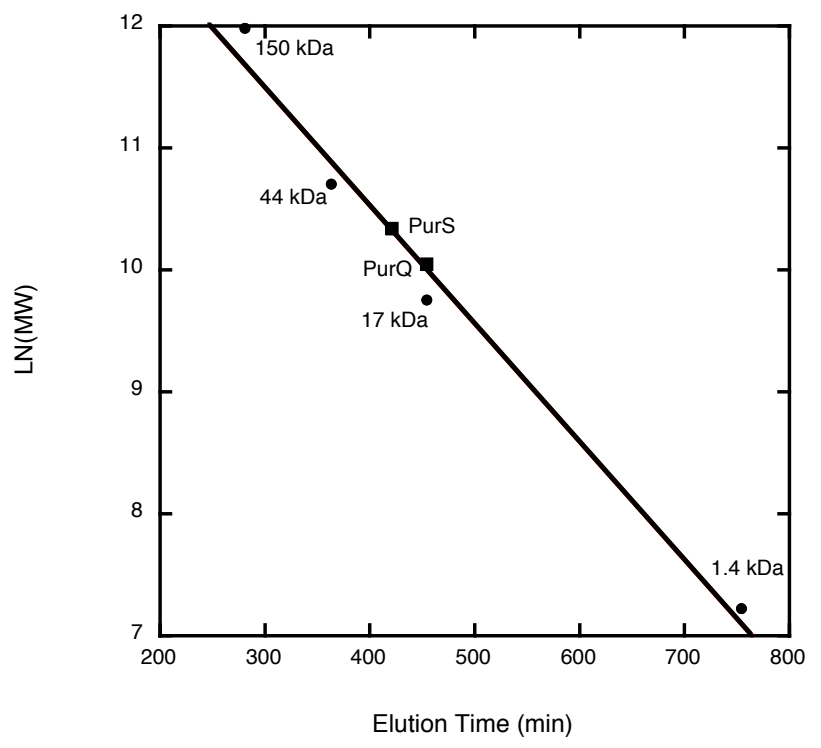

Supplemental Figure 9: S-200 HR SEC elution profile of PurS and PurQ. The elution times and MW of the standards are noted $(\bullet)$, and the best-fit line through the standards is drawn. PurS eluted at 424 min indicating a $30 \mathrm{kDa}$ species. PurQ eluted at $452 \mathrm{~min}$ indicating a $23 \mathrm{kDa}$ species. 


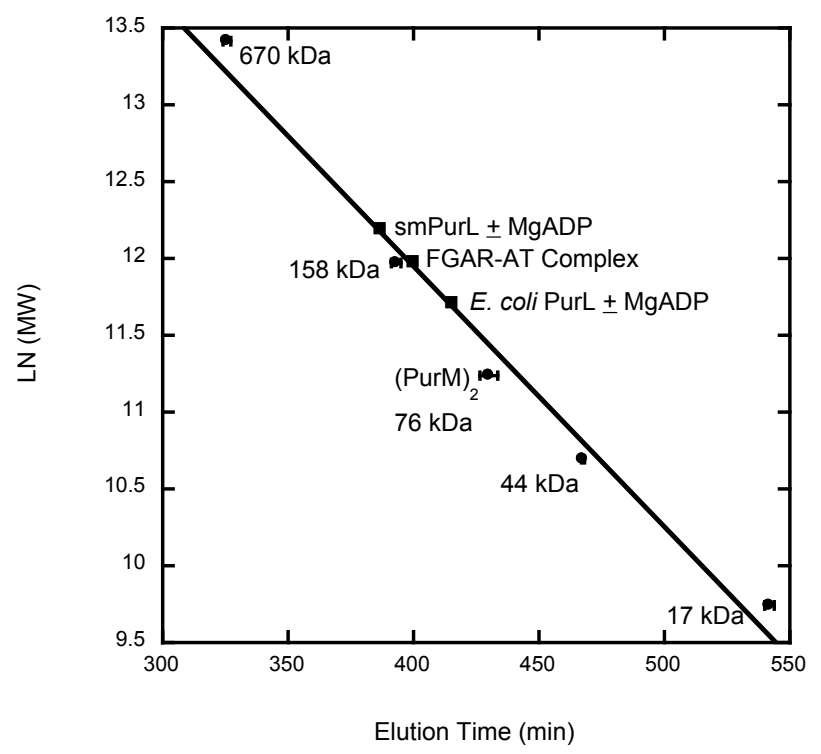

Supplemental Figure 10: SEC S-300 HR elution profile of smPurL and the FGAR-AT complex. The elution times and MW of the standards are noted $(\bullet)$ and the best-fit line through the standards is drawn. wt $E$. coli PurL eluted at 415 min (119 kDa) and smPurL eluted at $387 \mathrm{~min}(191 \mathrm{kDa})$ either in the presence or absence of MgADP. The FGARAT complex eluted at $400 \mathrm{~min}(153 \mathrm{kDa})$ in the presence of MgADP and glutamine. The standard deviation in the observed retention times ranged from 0.6 to $3.5 \mathrm{~min}(1.7-12$ $\mathrm{kDa}$ for proteins eluting near $190 \mathrm{kDa}$ ). 